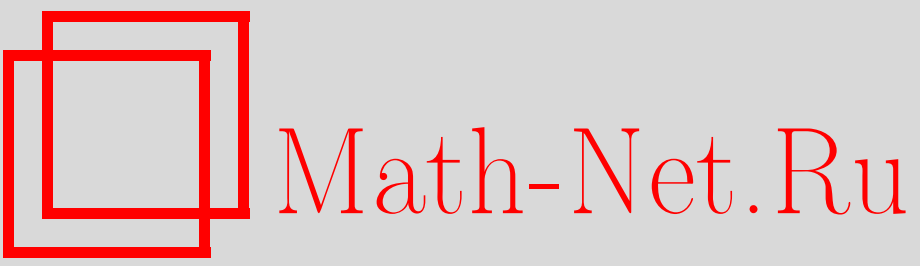

О. И. Мохов, Плоские пучки метрик и интегрируемые редукции уравнений Ламе, УМH, 2001, том 56, выпуск 2, 221-222

DOI: https://doi.org/10.4213/rm392

Использование Общероссийского математического портала Math-Net.Ru подразумевает, что вы прочитали и согласны с пользовательским соглашением

http://www . mathnet.ru/rus/agreement

Параметры загрузки:

IP : 18.207 .199 .55

26 апреля 2023 г., 10:24:42

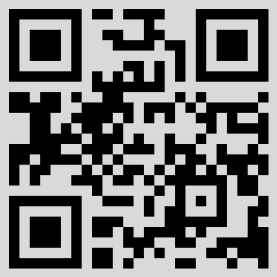




\title{
ПЛОСКИЕ ПУЧКИ МЕТРИК И ИНТЕГРИРУЕМЫЕ РЕДУКЦИИ УРАВНЕНИЙ ЛАМЕ
}

\author{
О.И. Мохов
}

В заметке проинтегрированы уравнения, описьвающие все неособые плоские пучки метрик. Напомним, что две псевдоримановы контравариантные метрики $g_{1}^{i j}(u)$ и $g_{2}^{i j}(u)$ назьваются согласованными, если для любой линейной комбинации этих метрик $g^{i j}(u)=\lambda_{1} g_{1}^{i j}(u)+\lambda_{2} g_{2}^{i j}(u)$, где $\lambda_{1}$ и $\lambda_{2}-$ произвольные константы, для которых $\operatorname{det}\left(g^{i j}(u)\right) \not \equiv 0$, коэффициенты соответствующих связностей Леви-Чивита и компоненты соответствующих тензоров римановой кривизны связаны тем же самым линейньм соотношением: $\Gamma_{k}^{i j}(u)=\lambda_{1} \Gamma_{1, k}^{i j}(u)+\lambda_{2} \Gamma_{2, k}^{i j}(u)$, $R_{k l}^{i j}(u)=\lambda_{1} R_{1, k l}^{i j}(u)+\lambda_{2} R_{2, k l}^{i j}(u)$ [1], [2]. В этом случае будем говорить также, что метрики $g_{1}^{i j}(u)$ и $g_{2}^{i j}(u)$ образуют пучок метрик. В случае, когда согласованные метрики $g_{1}^{i j}(u)$ и $g_{2}^{i j}(u)$ - плоские, т.е. $R_{1, k l}^{i j}(u)=R_{2, k l}^{i j}(u)=0$, произволшная линейная комбинация метрик $g_{1}^{i j}(u)$ и $g_{2}^{i j}(u)$ тоже является плоской метрикой, и легко видеть, что согласованность плоских метрик эквивалентна известному определению плоского пучка метрик или, другими словами, согласованной пары локальных невырожденных пуассоновых структур гидродинамического типа [3] (см. также [4]-[6], [8]). Две псевдоримановы метрики $g_{1}^{i j}(u)$ и $g_{2}^{i j}(u)$ назьваются неособой парой метрик, если собственные значения этой пары метрик, т.е. корни уравнения $\operatorname{det}\left(g_{1}^{i j}(u)-\lambda g_{2}^{i j}(u)\right)=0$, различны. Пучок метрик назьвается неособым, если он образован неособой парой метрик. Произвольная неособая пара метрик является согласованной тогда и только тогда, когда существуют локальные координаты $u=\left(u^{1}, \ldots, u^{N}\right)$ такие, что $g_{2}^{i j}(u)=g^{i}(u) \delta^{i j}$ и $g_{1}^{i j}(u)=f^{i}\left(u^{i}\right) g^{i}(u) \delta^{i j}$, где $f^{i}\left(u^{i}\right), i=1, \ldots, N$, - произвольные функции одной переменной (см. [1], [2]). Введем классические обозначения $g^{i}(u)=1 /\left(H_{i}(u)\right)^{2}$, $d s^{2}=\sum_{i=1}^{N}\left(H_{i}(u)\right)^{2}\left(d u^{i}\right)^{2}, \beta_{i k}(u)=\left(1 / H_{i}(u)\right) \partial H_{k} / \partial u^{i}, i \neq k$, где $H_{i}(u)-$ коэффициенть Ламе и $\beta_{i k}(u)$ - коэффициенты вращения.

ТЕОРема [1]. Произвольная неособая пара согласованных плоских метрик описывается следующей интегрируемой системой нелинейных уравнений, которая является редукцией уравнений Ламе:

$$
\begin{gathered}
\frac{\partial \beta_{i j}}{\partial u^{k}}=\beta_{i k} \beta_{k j}, \quad i \neq j, \quad i \neq k, \quad j \neq k \\
\frac{\partial \beta_{i j}}{\partial u^{i}}+\frac{\partial \beta_{j i}}{\partial u^{j}}+\sum_{s \neq i, s \neq j} \beta_{s i} \beta_{s j}=0, \quad i \neq j \\
f^{i}\left(u^{i}\right) \frac{\partial \beta_{i j}}{\partial u^{i}}+\frac{1}{2}\left(f^{i}\left(u^{i}\right)\right)^{\prime} \beta_{i j}+f^{j}\left(u^{j}\right) \frac{\partial \beta_{j i}}{\partial u^{j}}+\frac{1}{2}\left(f^{j}\left(u^{j}\right)\right)^{\prime} \beta_{j i}+\sum_{s \neq i, s \neq j} f^{s}\left(u^{s}\right) \beta_{s i} \beta_{s j}=0, \quad i \neq j,
\end{gathered}
$$

где $f^{i}\left(u^{i}\right), i=1, \ldots, N,-$ заданные произвольнье функции (собственные значения данной пары метрик).

Уравнения (1) и (2) - классические уравнения Ламе, а уравнения (3) определяют нетривиальные нелинейные дифференциальные редукции уравнений Ламе. Напомним метод Захарова интегрирования уравнений Ламе (1) и (2) (вариант метода обратной задачи рассеяния) [7]. Мы должны подобрать матричную функцию $F_{i j}\left(s, s^{\prime}, u\right)$ и решить линейное интегральное уравнение

$$
K_{i j}\left(s, s^{\prime}, u\right)=F_{i j}\left(s, s^{\prime}, u\right)+\int_{s}^{\infty} \sum_{l} K_{i l}(s, q, u) F_{l j}\left(q, s^{\prime}, u\right) d q .
$$

Работа вьполнена при финансовой поддержке Фонда Александра фон Гумболдта (Германия), а также РФФИ (грант № 99-01-00010) и INTAS (грант № 99-1782). 
Однопараметрическое семейство решений уравнений Ламе задается формулой

$$
\beta_{i j}(s, u)=K_{j i}(s, s, u) .
$$

В частности, если $F_{i j}\left(s, s^{\prime}, u\right)=F_{i j}\left(s-u^{i}, s^{\prime}-u^{j}\right)$, где $F_{i j}(x, y)$ - произвольная матричная функция двух переменных, то формула (5) дает решения уравнений (1). Чтобы удовлетворить уравнения (2), Захаров предложил наложить на функцию $F_{i j}\left(s-u^{i}, s^{\prime}-u^{j}\right)$ дополнительное линейное дифференциальное соотношение

$$
\partial F_{i j}\left(s, s^{\prime}, u\right) / \partial s^{\prime}+\partial F_{j i}\left(s^{\prime}, s, u\right) / \partial s=0 .
$$

Если функция $F_{i j}\left(s-u^{i}, s^{\prime}-u^{j}\right)$ удовлетворяет дифференциалному соотношению Захарова (6), то коэффициенты вращения $\beta_{i j}(s, u)(5)$ дополнительно удовлетворяют уравнениям (2). Чтобы разрешить линейные соотношения (6) для матричной функции $F_{i j}\left(s-u^{i}, s^{\prime}-u^{j}\right)$, можно ввести $N(N-1) / 2$ произвольных функций двух переменных $\Phi_{i j}(x, y), i<j$, и положить для $i<j$

$F_{i j}\left(s-u^{i}, s^{\prime}-u^{j}\right)=\frac{\partial \Phi_{i j}\left(s-u^{i}, s^{\prime}-u^{j}\right)}{\partial s}, \quad F_{j i}\left(s-u^{i}, s^{\prime}-u^{j}\right)=-\frac{\partial \Phi_{i j}\left(s^{\prime}-u^{i}, s-u^{j}\right)}{\partial s}$,

и, кроме того,

$$
F_{i i}\left(s-u^{i}, s^{\prime}-u^{i}\right)=\partial \Phi_{i i}\left(s-u^{i}, s^{\prime}-u^{i}\right) / \partial s,
$$

где $\Phi_{i i}(x, y), i=1, \ldots, N,-$ произвольные кососимметрические функции двух переменных: $\Phi_{i i}(x, y)=-\Phi_{i i}(y, x)($ см. [7]).

Лемма. Если две матричные функции, $F_{i j}\left(s-u^{i}, s^{\prime}-u^{j}\right) u$

$$
\widetilde{F}_{i j}\left(s-u^{i}, s^{\prime}-u^{j}\right)=\left(\sqrt{f^{j}\left(u^{j}-s^{\prime}\right)} / \sqrt{f^{i}\left(u^{i}-s\right)}\right) F_{i j}\left(s-u^{i}, s^{\prime}-u^{j}\right),
$$

обе удовлетворяют дифференциальному соотношению Захарова (6), то соответствующие коэффициенты вращения $\beta_{i j}(s, u)(5)$ удовлетворяют всем уравнениям (1)-(3).

Для $\widetilde{F}_{i j}\left(s-u^{i}, s^{\prime}-u^{j}\right)$ соотношение (6) дает $N(N+1) / 2$ линейных уравнений в частных производных второго порядка для $N(N+1) / 2$ функций $\Phi_{i j}\left(s-u^{i}, s^{\prime}-u^{j}\right), i \leqslant j$, двух переменных:

$$
\begin{gathered}
2 \frac{\partial^{2} \Phi_{i j}\left(s-u^{i}, s^{\prime}-u^{j}\right)}{\partial s \partial s^{\prime}}\left(f^{i}\left(u^{i}-s\right)-f^{j}\left(u^{j}-s^{\prime}\right)\right) \\
-\frac{\partial \Phi_{i j}\left(s-u^{i}, s^{\prime}-u^{j}\right)}{\partial s} \frac{d f^{j}\left(u^{j}-s^{\prime}\right)}{d s^{\prime}}+\frac{\partial \Phi_{i j}\left(s-u^{i}, s^{\prime}-u^{j}\right)}{\partial s^{\prime}} \frac{d f^{i}\left(u^{i}-s\right)}{d s}=0, \quad i \leqslant j .
\end{gathered}
$$

Любые решения линейных уравнений (10) порождают однопараметрические семейства решений системы (1)-(3) по формулам (7), (8), (4) и (5). Таким образом, наша задача линеаризована.

\section{СПИСОК ЛИТЕРАТУРЫ}

[1] O. I. Mokhov // math.DG/0005081. [2] О. И. Мохов // УМН. 2000. Т. 55. № 4. С. 217218. [3] B. Dubrovin // Lecture Notes in Math. 1996. V. 1620. P. 120-348; hep-th/9407018. [4] B. Dubrovin // Preprint SISSA 25/98/FM; math.DG/9803106. [5] E. V. Ferapontov // Topics in Topology and Mathematical Physics / ed. S. P. Novikov. Providence, RI: Amer. Math. Soc., 1995. Р. 33-58. [6] О. И. Мохов // Труды МИАН. 1999. Т. 225. С. 284-300. [7] V. Е. Zakharov // Duke Math. J. 1998. V. 94. P. 103-139. [8] Б. А. Дубровин, С. П. Новиков // Докл. АН СССР. 1983. Т. 270. № 4. С. 781-785.

Центр нелинейных исследований при Институте теоретической физики им. Л. Д. Ландау РАН, Принято редколлегией Department of Mathematics, University of Paderborn, Germany 01.02 .2001 E-mail: mokhov@genesis.mi.ras.ru, mokhov@landau.ac.ru; mokhov@uni-paderborn.de 